\title{
Resúmenes de Tesis
}

\section{Un experimento para Riemann. Introducción a la tesis convencionalista en la física relativista'}

\author{
MATÍAS DANIEL PASQUALINI ${ }^{2}$
}

La aparición de geometrías no euclidianas en siglo XIX llevó a algunos pensadores, como Poincaré, a considerar que los axiomas geométricos no son evidentes sino convencionales. Se puede usar una geometría u otra, haciendo convenientes ajustes en las leyes físicas. La elección de una geometría no es una cuestión que pueda estar sometida a control empírico, sino materia de simple conveniencia. Otros pensadores prefirieron pensar, como Gauss, que la cuestión de cuál es la geometría del mundo físico es una cuestión empíricamente decidible. Se sabe que Gauss concibió un arreglo experimental para proceder a dicha determinación: la medición de los ángulos internos de un triángulo formado por tres picos montañosos.

Poincaré había pronosticado que, aunque es posible hacer física con una geometría no euclidiana, nunca se abandonaría la euclidiana por ser la que permite las leyes físicas más simples posibles, las newtonianas. Sin embargo, la posterior aparición de la teoría de la relatividad por obra de Einstein (especial en 1905 y general en 1915) y su posterior confirmación empírica en 1919 pondrían en evidencia su desacierto. El hecho de que esté disponible una nueva física basada en la geometría riemanniana que ha sido empíricamente corroborada y que ha mostrado mayor poder explicativo que la física newtoniana construida sobre la geometría euclidiana replantea completamente el problema de Poincaré. ¿Constituye la física de Einstein algo así como un "experimento para Riemann" en el sentido en que la cuestión de la geometría del mundo físico se reduce a una cuestión empíricamente decidible como tantas otras? ¿O debemos interpretar el trabajo de Einstein como una geometrización de la física para sostener que el convencionalismo geométrico en sus aplicaciones físicas

I Tesina de Grado de la Licenciatura en Filosofía (Universidad Nacional de Rosario). Fecha de defensa: I4/I2/18. Director: Javier Castro Albano. Tribunal: Javier Castro Albano, Daniel Trapani y Sandra Lazzer.

Texto completo disponible en: http://hdl.handle.net/2/33//3867

2 Universidad Nacional de Rosario (Rosario, Santa Fe, Argentina). matiaspasqualini@gmail.com 
continúa vigente? La tesina de licenciatura Un experimento para Riemann (2018) procura poner a la luz cuáles son los principios de la física relativista para así hacerse cargo de estos interrogantes.

La primera respuesta que se dio a este interrogante pretendió mantener vigente el convencionalismo. Se produjo dentro de la esfera de influencia del Círculo de Viena. Reichenbach propuso una paradigmática reformulación de la física relativista en su célebre Philosophie der Raum-Zeit-Lehre (1928). El autor distingue entre unos principios a priori (la teoría filosófica de la relatividad) y unas leyes físicas a posteriori (la teoría física de la relatividad). Los principios a priori propuestos por el autor son fundamentalmente una definición de congruencia espacial y una definición de congruencia temporal. La primera definición fija ciertas condiciones en las cuales un cuerpo material pasa a ser considerado una regla que permita definir empíricamente la métrica del espacio. La segunda fija condiciones para hacer de un proceso físico periódico un reloj que permita definir la métrica del tiempo. Entre dichas condiciones se menciona la igualación de fuerzas universales a cero.

Aquel último punto no carece de importancia, ya que la introducción de hipotéticas fuerzas universales que distorsionen las distancias espaciales $y / 0$ las duraciones temporales era lo que permitía según Poincaré adaptar cualquier geometría a la data empírica. Estas definiciones permiten establecer una coordinación entre geometría y data empírica de manera de poder determinar la geometría del espacio-tiempo en base a simples mediciones. Las leyes físicas tendrían que adaptarse luego a la geometría del mundo físico así determinada. Lo curioso es que en la reconstrucción de Reichenbach el control empírico de la geometría del mundo físico depende de un conjunto de estipulaciones previas de carácter a priori convencional.

Debe hacerse notar aquí que el recurso de Reichenbach al convencionalismo no es inocente. En el contexto del Círculo de Viena, el convencionalismo se había convertido en una pieza clave de una nueva teoría semántica del a priori, en sustitución de la teoría transcendental del a priori kantiana. Para el positivismo lógico, no será admisible que los enunciados a priori sean a la vez sintéticos como pretendía Kant. Tal cosa contradice la asunción básica del empirismo según la cual no podemos obtener a priori información sobre el mundo. La semántica del positivismo lógico se apoyaba en dos principios interconectados. El reductivismo explica el modo en que los enunciados sintéticos significan: por una reducción de un enunciado general a enunciados inmediatamente observacionales. La verdad de estos enunciados será contingente y dependiente de la data empírica. Por otro lado, los enunciados a 
priori son verdaderos cualquiera sea la data empírica. Su carácter analítico convencional los hace universales y necesarios.

Reichenbach se había permitido trazar una distinción neta entre enunciados a priori convencionales y leyes físicas sobre el respaldo que le daba una semántica renovada y prometedora. Sin embargo, con el correr de las décadas se pusieron en evidencia dificultades insuperables. Quine se ocupó de objetar cada intento que Carnap hiciera de precisar la noción de analiticidad necesaria para explicar el carácter a priori de ciertos enunciados. Además, retomando una tesis de Duhem, atacó la posibilidad de que los enunciados sintéticos pudieran significar de modo aislado. Analiticidad y reductivismo serán denunciados como los últimos Dos dogmas del empirismo (1950). Se impondrá en adelante un holismo semántico bajo la forma de la tesis Duhem-Quine. El holismo de Duhem era uno meramente epistémico: no es posible corroborar una hipótesis aislada sino todo un conjunto de las mismas en virtud de la lógica de la falsación. La tesis Duhem-Quine es más audaz: un enunciado aislado no puede significar, sino el todo de una teoría o más bien el todo del lenguaje. Bajo la semántica holista, no será admisible distinción alguna entre enunciados a priori y sintéticos. La geometría y la física quedarán enteramente superpuestas.

No es difícil ver que en el contexto pospositivista no será sencillo defender un convencionalismo no trivial en física relativista. El holismo semántico no parece dejar margen para aislar enunciados a priori convencionales sobre los cuales se apoye el resto de la física. La prolija reconstrucción de Reichenbach queda en entredicho por apoyarse en una semántica inviable. Grünbaum intentará evadir la dificultad al proponer que el convencionalismo en física relativista no conecta con consideraciones semánticas sino directamente fácticas: el espacio y el tiempo mismos carecen de una métrica intrínseca y no puede ser sino asignada arbitrariamente. Argumenta en base al carácter continuo de los elementos que integran un intervalo espacial o temporal. Sin embargo, será la propuesta de una nueva teoría del a priori por parte de Friedman en Dynamics of Reason (200I) la que estimamos resuelve de modo superador el conflicto entre holismo y una eventual interpretación de la física relativista según la cual ésta depende de principios a priori. Se trata de la teoría del a priori relativizado.

De acuerdo a la teoría del a priori relativizado, la física relativista parte de principios que establecen de modo unívoco una coordinación entre geometría y data empírica. Sin embargo, estos principios no son definiciones de carácter analítico como pretendiera Reichenbach en su reconstrucción. Ni siquiera son estipulaciones completamente arbitrarias de respectivas 
métricas para espacio y tiempo como propone Grünbaum. Para Friedman, las reconstrucciones convencionalistas no hacen justicia a la auténtica estructura de la teoría de la relatividad. Cabe aclarar que aunque Friedman defiende que los principios de la física relativista son a priori en un sentido relevante, él rechaza que su postura se homologue a convencionalismo por razones que quedarán claras en lo que sigue. Para Friedman, entonces, tanto el principio de la relatividad especial como el de la relatividad general son proposiciones sintéticas (con contenido empírico) que adquieren los atributos de universalidad y necesariedad (tradicionalmente atribuidos a proposiciones a priori) no por el modo en que ellas en sí mismas están construidas sino por el modo en que se organiza el resto de la teoría a partir de ellas. Estas proposiciones resultarán a priori (un a priori relativizado) en el sentido en que su elevación a principio que determina de modo unívoco la estructura de la teoría no está determinada a posteriori sino por un factor decisional de quien diseña la teoría.

En relatividad especial, el principio a priori relativizado según Friedman es la constancia de la velocidad de la luz. Se trata de suyo de una proposición empírica bien corroborada por el experimento de Michelson-Morley. Einstein decidió tomar esta proposición que de suyo aparece como una simple contingencia empírica y decidió convertirla en una verdad necesaria en el contexto de toda una nueva teoría del espacio-tiempo que constituye la relatividad especial. Solo si excluye la posibilidad de una simultaneidad absoluta entre eventos y se pone en relación recíproca espacio y tiempo para considerar al espacio-tiempo como un mismo continuo cuatridimensional es que la constancia de la velocidad de la luz deja de ser una mera contingencia para pasar a estar investida de la necesariedad de un principio a priori. La constancia de la velocidad de la luz es asumida así como el único principio coordinativo de la relatividad especial. Einstein proyecta en la proposición empírica de la constancia de la velocidad de la luz todo un modo en el cual la geometría y la data empírica se coordinan para hacer posible una física. No hay una definición a priori para espacio y otra para tiempo como pretendieran los convencionalistas. En todo caso, las distancias espaciales y las duraciones temporales se ajustarán en función del estado de movimiento del observador al hecho de que la velocidad de la luz debe resultar constante cualquiera sea el estado de movimiento del observador. En relatividad especial tenemos entonces un espacio-tiempo de métrica euclidiana que se deduce del hecho de suponer a priori que la constancia de la velocidad de la luz es una ley (y por ello universal y necesaria) de la naturaleza.

Debemos insistir en el punto diciendo que la corroboración empírica del experimento de Michelson-Morley no forzó la adopción del espacio-tiempo de la relatividad especial. El 
mencionado experimento resultaba igualmente consistente con otras interpretaciones que mantenían el estatus meramente a posteriori y contingente de la velocidad de la luz salvando así el espacio y tiempo absolutos de la física newtoniana. La adopción de la constancia de la velocidad de la luz como un principio dependió de una determinación a priori. Se trata de un principio a priori que responde a la teoría del a priori relativizado de Friedman. Por tanto, no es un a priori analítico ni puramente convencional. También es una proposición sintética expuesta a contraejemplos. Señala Friedman que el hecho mismo de que el principio de la teoría tenga de suyo contenido empírico, no hace a la teoría más débil sino todo lo contrario. Un principio tal puede estructurar de modo unívoco el resto de la teoría. Un principio a priori meramente definicional (como los principios de la física newtoniana) estructura el resto de la teoría solo de modo parcial. La teoría no podría decidir entre múltiples modelos todos ellos empíricamente equivalentes. A saber, todos los modelos que se adecúan a las velocidades relativas observadas pero con velocidades absolutas diferentes son igualmente aceptables para la física clásica. La relatividad especial excluye por principio los modelos empíricamente equivalentes entre los que la teoría del espacio y tiempo de Newton no podía distinguir. Por ello mismo, aún siendo la relatividad especial empíricamente equivalente con una teoría que mantenga la simultaneidad absoluta y admita fuerzas que produzcan las distorsiones espaciotemporales necesarias para ser consistente con Michelson-Morley, debemos decir que la relatividad especial es superadora por contar con una estructura mejor determinada y con un potencial explicativo mayor. A saber, la relatividad especial es capaz de proponer una relación de equivalencia entre masa y energía que quedaba completamente por fuera del horizonte de la mecánica newtoniana.

Que el principio de la relatividad especial tenga contenido empírico y resulte simultáneamente a priori relativizado respecto a la misma teoría de la cual es principio, hace que a la vez su necesariedad no pueda ser demostrada por la misma relatividad especial (ya que es asumido a priori como su principio) sin embargo (dado que tiene contenido empírico) su necesariedad sí puede ser sometida a control empírico indirecto cuando la relatividad especial quede incluida dentro de una teoría de mayor generalidad. $Y$ ese es exactamente el caso respecto a la teoría de la relatividad general. Cuando la relatividad general consigue desplazar a la teoría de la gravitación newtoniana en la arena empírica (perihelio de Mercurio y experimento de Eddington en 19/9), el principio de la relatividad especial resulta empíricamente corroborado, ya que la relatividad general incluye la métrica del espacio-tiempo de la relatividad especial a escala infinitesimal o en ausencia de materia. Esta dinámica de la 
razón que permite que un principio a priori relativizado sea empíricamente controlado en el contexto de una teoría de mayor generalidad, es la que da título a la mencionada obra de Friedman.

Un razonamiento análogo al que hemos planteado respecto al principio de la relatividad especial es igualmente aplicable al principio de la relatividad general: el principio de equivalencia entre masa inercial y gravitacional. Omitiremos en este resumen el detalle del mismo, remitimos al lector interesado a la mencionada tesina. Solo mencionaremos que las ecuaciones de campo de la relatividad general ponen al tensor métrico del espacio-tiempo en función de la distribución local de masa y energía. Esto es muy significativo ya que, contrariamente a lo que alguna especulación convencionalista ha supuesto, la relatividad general no sustituye elementos físicos por geométricos sino que más bien pone a los elementos geométricos en entera dependencia de los físicos.

Por último, corresponde señalar que hemos encontrado en la teoría del a priori relativizado de Friedman una potencial teoría semántica compatible con el holismo epistémico de Duhem. La semántica contenida en la teoría del a priori relativizado equidista tanto del atomismo semántico del positivismo lógico en que el enunciado aislado era capaz de producir significado, como del holismo semántico de Quine en que la unidad mínima de significado es virtualmente la totalidad de un lenguaje natural. La coordinación establecida en los principios a priori de una teoría científica (el ejemplo más claro es la física) es condición de posibilidad del significado empírico de las demás proposiciones de la teoría. Sin la coordinación, la cuestión de la adecuación empírica de las proposiciones que dependen de ella no podría decidirse. El significado aquí se produce por el particular modo en que quedan interrelacionados los principios a priori y las demás proposiciones de la teoría. El significado está de alguna manera codificado en la estructura interna de la teoría.

La tesina concluye reconociendo que la tesis convencionalista no es adecuada para dar cuenta de la física relativista, en atención al carácter de suyo sintético de sus principios. Esto no nos impide distinguir en ella una estructura entre principios a priori relativizados y proposiciones propiamente empíricas. La teoría del a priori relativizado aplicada a la física relativista arroja como resultado que sí es posible concebir "un experimento para Riemann". En cierto sentido, Michelson-Morley y cualquier corroboración empírica del principio de equivalencia, en el contexto de la teoría de la relatividad, constituyen un experimento tal. 
MATÍAS DANIEL PASQUALINI

Un experimento para Riemann

Resúmenes de Tesis

\section{Bibliografía}

Friedman, M. (200I). Dynamics of Reason. CSLI Publications.

Quine, W. V. (2005). Dos dogmas del empirismo. En L.M. Valdés Villanueva (ed.). La búsqueda del significado (Pp. 145-267). Tecnos.

Reichenbach, H. (1957). The Philosophy of Space and Time. Dover. 